\title{
VULNERABILIDAD COGNITIVA: UNA MIRADA DESDE EL MODELO COGNITIVO-CONDUCTUAL
}

\author{
Mavenka Cuesta Guzmán ${ }^{1}$ \\ Karen Villacob Fernández²
}

\section{Resumen}

El presente capítulo pretende explicar el concepto de vulnerabilidad desde el modelo cognitivo-comportamental, haciendo una revisión de los distintos modelos teóricos que han intentado explicar su génesis y forma de funcionamiento para comprender la adquisición de patologías clínicas, como ha sido el caso de la depresión. La vulnerabilidad, como aquel factor que predispone al individuo al desarrollo de trastornos psicológicos, tiene una base biológica y psicológica, siendo esta última la que se abordó en el presente capítulo, entendiéndose como vulnerabilidad cognitiva, la cual inicia su formación a partir de la confluencia de diversos factores como el temperamento, el estilo de crianza, las experiencias tempranas y el sistema informacional individual. Estos factores confluyen en la formación de componentes cognitivos, denominados en la literatura como esquemas o creencias centrales, sistemas de procesamiento informacional, sesgos, errores de pensamiento, entre otros, que una vez instalados predisponen al individuo al malestar emocional al ser activados por sucesos llamados estresores. Es así, como la vulnerabilidad cognitiva no es un factor responsable de las patologías, pero sí predispone al sujeto a padecerlas, dependiendo de la relación que guarden el contenido del factor estresor con el contenido del esquema cognitivo aprendido. Una patología, donde mayormente ha sido estudiada la vulnerabilidad cognitiva, ha sido en la depresión.

Palabras claves: Vulnerabilidad, componentes cognitivos, trastornos psicológicos, depresión.

$1 \quad$ Magister en Psicología. Psicóloga clínica, Docente en la Universidad Simón Bolívar, Barranquilla-Colombia. E-mail mavenkac@gmail.com

2 Magíster en Psicología. Psicóloga clínica, Psicoterapeuta, Docente en la Universidad Simón Bolívar, Barranquilla-Colombia. E-mail: kvillacob@hotmail.com. 


\section{Abstract}

This chapter aims to explain the concept of vulnerability from the cognitive-behavioral model, making a review of the different theoretical models that have tried to explain their genesis and way of functioning to understand the acquisition of clinical pathologies, as has been the case of depression. Vulnerability, as the factor that predisposes the individual to the development of psychological disorders, has a biological and psychological basis, the latter being addressed in this chapter, understood as cognitive vulnerability, which begins its formation from the confluence of various factors such as temperament, parenting style, early experiences and the individual information system. These factors converge in the formation of cognitive components, denominated in the literature as central schemes or beliefs, informational processing systems, biases, thinking errors, among others, which once installed predispose the individual to emotional distress when activated by events called stressors This is how cognitive vulnerability is not a factor responsible for pathologies, but it does predispose the subject to suffer them, depending on the relationship between the content of the stressor factor and the content of the cognitive scheme learned. A pathology, where cognitive vulnerability has mostly been studied, has been in depression.

Keywords: Vulnerability, cognitive components, psychological disorders, depression.

\section{Introducción}

El concepto de vulnerabilidad, inicialmente, ha sido estudiado desde el modelo cognitivo-conductual para el estudio de la depresión y, posteriormente, al estudio de otras patologías.

De la misma manera como desde la medicina se ha utilizado el modelo Diátesis-Estrés para estudiar aquellos factores que predisponen a un individuo al desarrollo de una enfermedad, como también a todos aquellos posibles factores estresores que la activan, asimismo, la psicología clínica, desde el modelo cognitivo comportamental, en la explicación de cómo se forman las patologías, se ha interesado en estudiar todos aquellos factores que coadyuvan en la aparición y mantenimiento de los trastornos psicológicos. A aquellos factores que hacen al individuo susceptible de padecerlos se le reconoce como vulnerabilidad, lo que en el modelo médico 
representaría la diátesis. La vulnerabilidad integra aspectos biológicos, psicológicos y sociales, correspondientes a facilitar o predisponer al individuo a una variedad de patologías (Riso, 2009).

La vulnerabilidad que abordaremos en el presente capítulo, hace mención a la vulnerabilidad psicológica, aquella que ha logrado definirse como un constructo útil para referirse a características internas, latentes y estables de una persona que pueden facilitar la aparición de síntomas depresivos ante la presentación de estresores vitales en un dominio central que guarde relación con la autovalía en el caso de la depresión (Ingram, Miranda, \& Segal, 1998).

Nótese bien que se define como características que "pueden" facilitar aparición de síntomas, lo cual nos indica que no es el único factor responsable de la existencia de las patologías clínicas.

Esta vulnerabilidad psicológica no es directamente observable, pero se hace evidente a partir de eventos que lo activen, lo cual explica la afirmación de Ingram et al. (1998), que, para que esta vulnerabilidad predisponga a la aparición de diversos trastornos psicológicos, se requiere de un evento estresor o, también llamado en la literatura, factor precipitante.

El factor precipitante representa un evento que, al aparecer en la vida del individuo, podría activarle la vulnerabilidad latente que se ha desarrollado tiempo atrás.

De este modo cabe preguntarse ¿Qué hace que un individuo sea más sensible a que se active su vulnerabilidad, mientras que otros podrían mostrarse menos vulnerables a pesar de los estresores que enfrentan? ¿Qué características debe tener el evento estresor para tener la capacidad de activar la vulnerabilidad en el individuo?

Para comprender la interacción de ambos factores, en el desarrollo de trastornos psicológicos, es importante resaltar la existencia de una vulnerabilidad cognitiva.

\section{Vulnerabilidad cognitiva}

La vulnerabilidad cognitiva consiste en una predisposición cognitiva conformada por un sistema de creencias o contenidos esquemáticos acerca 
de sí mismo y del mundo, que el individuo ha desarrollado a lo largo de su vida y que pueden mantenerse inactivos por un tiempo.

Este sistema de creencias que el individuo desarrolló, al ser disfuncionales, se constituyen en la base sobre la cual los trastornos psicológicos aparecen. Esto no quiere decir que esta vulnerabilidad obliga al trastorno a aparecer, pero sí establece la base para que ocurra, si ciertos eventos estresantes que guardan correlación informacional con la creencia o esquema llegan a presentarse (Riso, 2009).

Dependiendo de qué tanto los estresores guarden relación con el contenido esquemático, la vulnerabilidad cognitiva se activará. Sin embargo, mientras la activación no se dé o los estresores no guarden esa relación, los auto esquemas permanecen estables.

En este sentido, la psicopatología aparece cuando confluyen la vulnerabilidad cognitiva y el evento estresor que pone en jaque la creencia del paciente.

Es así, como algunas personas, al desarrollar un sistema de creencias ilógicas y disfuncionales, aumentan la probabilidad de ser susceptibles a la aparición de trastornos, y a esto se le llamó vulnerabilidad cognitiva.

\section{Componentes de la vulnerabilidad cognitiva}

Un componente cognitivo de la vulnerabilidad consiste en desarrollar una percepción distorsionada de sí mismo frente a los eventos, como ocurre, por ejemplo, en los trastornos de ansiedad, en donde el individuo se percibe poco suficiente o con poco control interno y externo sobre los peligros. De la misma manera, para el caso de la depresión, se manifiestan pensamientos auto referenciales negativos de sí mismo, del mundo y del futuro que, al enfrentarse el individuo a estresores negativos significativos congruentes con sus cogniciones depresogénicas dominantes, se hacen vulnerables a padecer el trastorno (Londoño, Jiménez, Juárez, \& Marín, 2010).

Igualmente, se ha encontrado la existencia de esta vulnerabilidad en otros trastornos como fobias, abuso de sustancias, entre otros.

Existen componentes cognitivos importantes que influyen a que el individuo desarrolle esa percepción distorsionada de sí mismo y del mundo, 
los cuales se explican a continuación. Estos componentes cognitivos están conformados por los esquemas o creencias centrales, el procesamiento informacional y las estrategias de afrontamiento.

\section{Los esquemas}

Beck (2012) define el esquema cognitivo como una estructura cognitiva que selecciona, codifica, y evalúa los estímulos que afectan al organismo. El esquema hace que el individuo se vuelva selectivo con la información proveniente del ambiente, dependiendo de su contenido.

Si el contenido del esquema se forma a partir de vivencias negativas y las creencias sobre hechos falsos, el esquema resultante será disfuncional. Un esquema disfuncional, a su vez, generará el desarrollo de pensamientos y conductas maladaptativas (Riso, 2009).

Por su parte, Young, Klosko y Weishaar (2013) identificaron la presencia de esquemas disfuncionales en trastornos crónicos, los cuales denominó esquemas maladaptativos tempranos, y los define como estructuras extremadamente estables y duraderas, elaboradas durante la infancia, que se desarrollan y fortalecen durante toda la vida y son disfuncionales. Estos esquemas sesgan la forma como el individuo percibe el mundo, lo interpreta y se enfrenta a él, debido a que son considerados a priori como verdades absolutas y rígidas. Son multifacéticos, contienen componentes cognitivos, afectivos, interpersonales y conductuales, y están por fuera de la conciencia.

Los esquemas maladaptativos tempranos se forman en edades tempranas gracias a la influencia de diversos factores, entre los cuales se encuentran el temperamento, el apego y las experiencias tempranas. De esta manera, la confluencia de estos factores, predisponen a la formación de los esquemas maladaptativos, los cuales han sido clasificados por Young et al. (2013) en esquemas de abandono, abuso, vulnerabilidad al daño, deprivación emocional, incompetencia, entro otros, que pueden predisponer al individuo al desarrollo de trastornos psicológicos crónicos.

Veamos el ejemplo de una persona cuyo temperamento marca su tendencia a responder a ciertos de hechos de forma específica. Podría ser el caso de un niño con temperamento nervioso (Kagan $\&$ Snidman, 2004). Este niño, de alguna manera, se muestra sensible a las experiencias 
novedosas, y sus padres, al verlo reaccionar ante los estímulos externos de esa manera, optan por sobreprotegerlo, inhibiendo el desarrollo de sus habilidades sociales. Al enfrentarse este niño en su ámbito escolar, existe una alta probabilidad de que sea susceptible a experiencias de rechazo y/o acoso escolar, por no tener las competencias relacionales que le faciliten una integración social favorable. Este niño, frente a toda esta predisposición anterior, cuenta de base con elementos que facilitaría la formación de un posible esquema de incompetencia, que podrían hacerlo más vulnerable que otros a sufrir algún tipo de trastorno psicológico en etapas posteriores de su vida.

Frente a lo anterior, podemos concluir que el desarrollo de esquemas cognitivos maladaptativos tempranos, representan un componente fundamental en el desarrollo de la vulnerabilidad cognitiva.

Al igual que Young, Beck, en su modelo de terapia cognitiva de la depresión (Beck, 2012), había desarrollado el concepto de creencia central para explicar los trastornos psicológicos cuando se establecen de forma disfuncional. Beck sostiene que las creencias centrales son ideas tan fundamentales y profundas que no se suelen expresar, ni siquiera ante uno mismo, y son consideradas por la persona como verdades absolutas (Beck, 2000). En la literatura cognitivo-conductual, tanto el esquema cognitivo como la creencia central son categorizadas en el mismo nivel de profundidad informacional y son considerados factores de vulnerabilidad cognitiva en la psicopatología.

\section{Procesamiento informacional}

El esquema o la creencia no operan solos; funcionan con un procesamiento selectivo de la información. Este se encarga de guiar, seleccionar, filtrar las experiencias nuevas.

Cuando la creencia central o el esquema no son adaptativos, el procesamiento informacional selecciona en el ambiente aquello que termine por reforzar el contenido de la creencia, volviéndose un proceso de ahorro cognitivo que economiza los recursos cognitivos a la hora de atender e interpretar los hechos (Riso, 2009).

Una vez un individuo ha creído en algo sobre él o el mundo, intentará mantenerlo seleccionando aquella información que lo confirme, 
y no seleccionará aquella que la contradiga. Esta selectividad informacional ocurre gracias a una serie de procesos como los sesgos, otros tipos de creencias disfuncionales denominadas creencias intermedias (Beck, 2000) y errores de pensamiento que distorsionan la forma como el individuo atiende, percibe y comprende el mundo.

Este tipo de procesamiento informacional se basa en un procesamiento automático de la mente, no consciente, rápido, con poco esfuerzo, y que favorece a una mayor probabilidad de errores (Langer, 1978, citado en Riso, 2009)

Es así, como en el caso de una persona con trastorno de ansiedad, el contenido informacional de su esquema le hace atender de manera selectiva a la información de amenaza que se encuentra en el ambiente, interpretándolo como peligroso, haciéndole desplegar una serie de estrategias o maniobras de solución frente al problema, que, en el caso de la ansiedad, la mayoría de las veces opera con conductas de evitación y/o huida.

De manera similar, para el caso de la depresión, la selectividad informacional apuntará la mayor parte del tiempo hacia sus debilidades o hacia los eventos negativos, dando origen a errores de pensamiento, denominadas por Aarón Beck como distorsiones cognitivas, en donde habrá la tendencia a minimizar sus cualidades y competencias favorables y maximizar las dificultades o experiencias no tan favorables, provocando estados de ánimo bajos, pesimismo y aislamiento, entre otros síntomas comúnmente presentes en la depresión.

La depresión ha sido la patología donde más se ha estudiado la vulnerabilidad cognitiva y se verá en detalle más adelante.

\section{Estrategias de afrontamiento}

Una vez el sistema informacional ha operado a través de los diferentes procesos, confirmando la creencia central en el individuo, genera en él respuestas de acción, las cuales, de alguna forma, obedecen al modo como se procesó la información, dando como resultado comportamientos disfuncionales. Esta disfuncionalidad en el comportamiento provoca respuestas en el ambiente, que se convierten en nuevos elementos confirmatorios del esquema de la persona, envolviéndola en un círculo de 
retroalimentación esquemática o autoperpetuación (Pretzer \& Beck, 2004, citados en Riso, 2009)

A estos comportamientos disfuncionales se les llamó, en la terapia cognitiva, estrategias de afrontamiento desadaptativos (Young et al., 2013), los cuales operan como sistemas de seguridad que pretenden disminuir o modificar el problema o hacen que el paciente se proteja así mismo del sufrimiento que le puede generar si se activa su esquema disfuncional (Riso, 2009).

Entre las estrategias de afrontamiento mal adaptativas, destacadas por los modelos de terapia cognitiva, se encuentran las estrategias de evitación, compensación y mantenimiento esquemático. (Young et al., 2013; Beck, 2000) Las tres tienen formas distintas de desenvolverse ante los estímulos presentes, más cumplen la misma función de proteger y mantener el esquema disfuncional evitando su activación. Recordemos que esta activación es lo que, desde el modelo cognitivo conductual, generaría el malestar emocional y los síntomas clínicos.

De lo anteriormente expuesto, queda claro que, mientras existan esquemas disfuncionales formados desde temprana edad, estos guiarán al sujeto a seleccionar información proveniente del exterior, o, en algunos casos, del interior, tal como ocurre en los trastornos de pánico, que al ser filtrada e interpretada de manera sesgada, conforme al contenido esquemático, provocarán en el sujeto comportamientos disfuncionales o estrategias que, a su vez, generarán experiencias en el ambiente, que, al recibirlas nuevamente, serán procesadas de forma errónea, confirmando, así, los contenidos esquemáticos subyacentes del sujeto.

Estos componentes forman parte de un sistema de autoperpetuación cognitiva o retroalimentación esquemática, del cual el sujeto no es consciente y lo mantiene porque le hace experimentar alivio al no permitir que su esquema maladaptativo se active, pudiendo exacerbar los síntomas de su patología.

En ese orden de ideas, la vulnerabilidad cognitiva se compone de diferentes elementos, procesos y estrategias que pueden estar operando sin que el individuo lo sepa, o bien pueden estar inactivos, hasta que un estresor congruente con la información del esquema o la creencia, lo haga activar. 


\section{Vulnerabilidad cognitiva en la depresión}

La vulnerabilidad cognitiva es fundamental para la compresión y descripción de diversas patologías psicológicas en el ser humano. La forma en cómo procesa el individuo la información ante los estímulos, las distorsiones cognitivas y las creencias nucleares, favorecen la génesis de varios trastornos psicológicos, tal como ocurre en la depresión. La perspectiva cognitiva ha sido muy útil para dar explicaciones en el campo de la depresión. Además de tener tratamientos eficaces para su intervención, también permite tener mejor comprensión de los aspectos que influyen en la génesis de la depresión en el ser humano.

En este apartado del presente capítulo se describirán diversos modelos teóricos que explican aquellos aspectos que son considerados vulnerables para el origen de un trastorno depresivo.

En primera instancia, es relevante conocer que, de acuerdo con el Ministerio de Salud y Protección Social en Colombia, existe una prevalencia de un $12,1 \%$ y $13,1 \%$ de la población con depresión, lo que hace importante tener un adecuado conocimiento de los factores que facilitan su desarrollo, para así lograr una compresión amplia que favorezca niveles de intervención óptimos en la práctica clínica (Vanegas et al., 2017).

\section{Modelos explicativos}

Para dar explicación al concepto de vulnerabilidad cognitiva en el trastorno depresivo, Abramson, Seligman y Teasdale (1978) plantean un modelo que ellos denominaron indefensión aprendida, el cual argumenta que, cuando el individuo experimenta diversas situaciones aversivas, evidencia un estilo atribucional a causas internas, globales y estables, lo cual lo hace sentir y percibirse a sí mismo de forma indefensa y con pocas estrategias para afrontar de forma favorable dichas situaciones.

De igual manera, concibe que, en un futuro, el individuo tampoco tendrá una contingencia entre sus respuestas (cognitivas, emocionales y conductuales) y sus consecuencias, facilitando déficits cognitivos, emocionales y motivacionales.

De acuerdo con lo anterior, la indefensión aprendida le brinda importancia a los procesos cognitivos que realiza el ser humano, como 
determinante en el desarrollo de los trastornos depresivos. Asimismo, a las experiencias previas que haya tenido, siendo situacionales o específicas. Es así como los seres humanos aprenden un estilo cognitivo, y se evidencia cuando experimenta situaciones que percibe incontrolables, influyendo en un déficit en la resolución de los problemas, acompañado con una inadecuada identificación entre su comportamiento y sus consecuencias, desarrollando, así, expectativas negativas respecto al futuro.

Por lo tanto, desde el modelo cognitivo conductual es relevante para la comprensión de la génesis de un trastorno depresivo, revisar la manera en que las personas le brindan significado e interpretan sus experiencias, como también qué tan vulnerable ha sido a episodios depresivos de forma recurrente. Por su parte, Beck y Alloy coinciden en el papel que juega la vulnerabilidad cognitiva como factor de riesgo para sufrir depresión, y cómo interviene en el procesamiento de la información ante los sucesos de gran importancia para la persona (Alloy et al, 1999 citado por Martínez, 2011).

Por otra parte, Ingram et. al (1998) sostienen que la depresión se presenta a raíz de la confluencia entre aspectos cognitivos y situaciones de estrés, por lo cual, si el individuo no experimenta situaciones estresantes, no se podrá diferenciar de una persona que posea una vulnerabilidad cognitiva de aquella que si la tendría, es decir, que la única forma de que se active un patrón depresogénico en el sujeto es si se enfrenta a un evento aversivo, activándose patrones cognitivos de forma negativa, permitiendo una predisposición al trastorno. Pero si, por el contrario, el evento estresante se le presenta a una persona no vulnerable, es probable que sus síntomas no cumplan con criterios para una depresión.

El modelo de Aaron Beck ha sido un referente en la psicología cognitiva, explicando el concepto de vulnerabilidad en la depresión. Según Beck, las personas que experimentan depresión poseen una serie de errores cognitivos, producto de un procesamiento de información inadecuado que generan pensamientos negativos, y estos se encuentran apoyados por unas creencias centrales, por lo general, de minusvalía e indefensión, permitiendo la presencia de un proceso en donde el individuo desarrolla una visión negativa de sí mismo, de los demás y del futuro, que él denominó triada cognitiva (Ingram et al, 1998). 
Beck (2012) sostiene que las experiencias tempranas en niños que han sido víctimas de rechazo y crítica por parte de sus padres, generan esquemas disfuncionales relacionados con percepciones de sí mismo de minusvalía, siendo estas causas más distales para el desarrollo de una depresión. Por otro lado, también argumenta que situaciones más cercanas al individuo facilitan la aparición de estados depresivos, como puede ser una situación difícil de manejar, la activación de los esquemas disfuncionales ya aprendidos en la infancia y, como consecuencia de esto, aparecerán los pensamientos negativos que se encuentran inmersos en la triada cognitiva mencionada anteriormente.

La teoría del apego, igualmente, ha sido considerada un modelo explicativo de la vulnerabilidad, que facilita la génesis de la depresión. De acuerdo con esto, Hammen (1991) argumenta que el apego inseguro se encuentra relacionado con respuestas depresivas intensas ante situaciones vitales negativas. Y plantea que infantes que han sido víctimas de abuso verbal con descalificaciones personales, son también propensos a desarrollar esquemas cognitivos con tendencia a la autoinculpación y, por lo tanto, a sufrir con mayor probabilidad episodios depresivos (Alloy et al., 2009, citado por Vázquez, Herves, Hernangómez y Romero, 2010).

Siguiendo esta línea, Ingram et. al (1998) coinciden en que el apego es un aspecto importante para el desarrollo de la depresión, pero lo resaltan con el concepto de modelos internos de trabajo. Sostienen que las representaciones cognitivas que internalizan los seres humanos, en cuanto a las primeras relaciones que tengan con las figuras de apego, serán de suma importancia en las expectativas que tengan para el resto de su vida. Por lo tanto, los niños que experimenten tipos de apego inseguros, evidenciarán modelos internos de trabajo con un procesamiento de la información distorsionada en las relaciones interpersonales que tuvieron con sus figuras tempranas, facilitando, en un futuro, el establecimiento de relaciones personales desadaptativas.

Por otra parte, el estrés también ha sido relevante en la teoría cognitiva, para brindar explicaciones para el desarrollo de la depresión. Ingram et al. (1998) plantean que el estrés representa aquellos acontecimientos vitales o importantes que perturban las estabilidad emocional, cognitiva y fisiológica del individuo, alterando, así, sus estrategias de afrontamiento. Las investigaciones y modelos teóricos, que han establecido relaciones 
entre los sucesos estresantes y la depresión, han centrado su atención en los sucesos estresantes episódicos, siendo de carácter negativo e indeseable, concluyendo que, tanto el número de los episodios como la gravedad de éstos, tienen una estrecha relación con el inicio de un episodio depresivo. Sin embargo, estudios indican que acontecimientos estresantes con baja intensidad, pero que se suelen presentar de forma recurrente, son más prevalentes que los sucesos mayores, dando mayor explicación en el desarrollo de los trastornos depresivos. (McGonagle \& Kessler, 1990; Turner, Wheaton \& Lloyd, 1995, citados por Vázquez, et al., 2010).

Los sesgos cognitivos, de igual manera, son considerados importantes para explicar la vulnerabilidad cognitiva la depresión, ya que diversos estudios avalan que los sesgos cognitivos tienen presencia en los trastornos emocionales. (Mathews \& MacLeod, 2005, citado por Duque, López, Blanco \& Vásquez, 2015). En este orden de ideas, Beevers (2005) sostiene que los pensamientos negativos que evidencian los sujetos con depresión, manifestándose como recuerdos intrusos o sesgos de atención, pueden generar estados de ánimo negativos, y, si estos no son intervenidos de forma adecuada, pueden facilitar cada vez más que el individuo realimente sus procesos cognitivos permitiendo el desarrollo del trastorno.

De acuerdo con esto, los sesgos de memoria y de atribuciones causales han sido los más estudiados y contrastados de forma empírica. En relación con la memoria, los modelos cognitivos de la depresión se han interesado en conocer qué relación existe entre la depresión y/o los estados afectivos y los procesos de memoria, puesto que los pacientes diagnosticados con depresión en la práctica clínica suelen verbalizar dificultades en su memoria.

En este orden de ideas, Matt, Vázquez y Campbell (1992) encontraron, mediante un metaanálisis que realizaron, que las personas con depresión suelen recordar más información negativa que positiva. Por otro parte, Williams et al., (2007) planteaban que los sujetos con depresión evidencian recuerdos autobiográficos de su vida, puesto que tienden a recordar de forma generalizada y vaga solamente vivencias negativas de sus propias vidas, verbalizando expresiones como: "mi vida ha sido mala", "solo he tenido sinsabores", "nunca he disfrutado de nada".

Con respecto a las atribuciones causales, las teorías atribucionales han tenido impacto en las investigaciones para explicar el desarrollo de la 
depresión, como es el caso de la teoría de la indefensión aprendida y de la desesperanza, por Abramson et al., (1978), la cual argumenta que los seres humanos, cuando atraviesan una situación aversiva, intentan saber por qué se ha generado, brindando explicaciones causales, por lo general, de forma depresiva (Vázquez, Hervas, Hernangómez, \& Romero, 2010).

Por tanto, estas personas evidencian un estilo atribucional interno, global y estable ante sucesos negativos, facilitando, así, reacciones depresivas ante situaciones estresantes (Vázquez, Díez-Alegría, Hernández-Lloreda, $\&$ Nieto, 2008). Es decir, cuando la persona suele explicar los diversos sucesos negativos y/o de fracaso que ha experimentado a través de su vida, por medio de causas dirigidas hacia sí mismo, como por ejemplo, su falta de recursos, o porque se percibe incompetente y, además, piensa que estas causas se mantendrán en el presente y en el futuro, logrando afectar sus áreas de vida —como familiar, laboral, etc.—, existirá, entonces, mayor probabilidad de tener síntomas depresivos en el momento en que tenga que enfrentarse a estas situaciones, percibidas por la persona como relevantes.

Abela (2002) afirma que existen estudios que han demostrado que la prevalencia de síntomas depresivos son el resultado de la relación entre la intensidad de las situaciones negativas y el estilo atribucional que realizan los participantes; en otros casos, la baja autoestima también se denominó un tercer factor predictor para la presencia del trastorno. Otra línea de investigación ha mostrado la capacidad que tiene el individuo para realizar diferentes atribuciones, sean estas internas o externas, globales o específicas, dependiendo de las particularidades del acontecimiento. Esto es lo que Moor y Fresco (2007) denominan flexibilidad atribucional, puesto que ellos sostienen que las personas pueden evidenciar una rigidez o flexibilidad en su estilo atribucional, ante las situaciones vitales en las que se encuentren.

De acuerdo con lo anterior, estos autores sostienen que la depresión se desarrolla por la relación existente entre las experiencias negativas y la poca flexibilidad atribucional, siendo, entonces, considerado un factor de vulnerabilidad para el trastorno depresivo. Por otro lado, también se ha encontrado que lo permite la presencia de una sintomatología depresiva. No es el estilo atribucional, como tal, sino la flexibilidad atribucional que el individuo realice (Fresco, Rytwinky, \& Craighead, 2007). 
Con respecto a la atención, autores como Whitmer y Gotlib (2013) plantean un modelo de amplitud atencional, donde argumentan que en la depresión se evidencia en el individuo disminución de procesos atencionales y, por lo tanto, disminuirán los repertorios de pensamientos y acciones, influyendo en la creación de espirales emocionales negativas. De acuerdo con esto, los procesos cognitivos elementales, como lo es la atención, se encuentran relacionados con estructuras y circuitos ubicadas en el cerebro, los cuales podrían estar unidos a procesos más complejos de feedback, desarrollando consecuencias emocionales y comportamentales propias de la depresión (De Raedt \& Koster, 2010; Gotlib \& Joormann, 2010; Vázquez et al., 2010).

Por último, la rumiación mencionada anteriormente, también ha sido tenida en cuenta como factor explicativo en los procesos depresivos. El estilo rumiativo (Nolen-Hoeksema, 1991, citado por Inoñán, \& Cassaretto, 2011), lo define como un estilo de pensamiento y/o tendencia que tiene el sujeto de centrarse de forma extrema en las emociones negativas y, a partir de esto, buscar constantemente las causas y consecuencias de su estado, para así llegar a una comprensión.

El modelo rumiativo, de igual manera, plantea que el individuo evidencia diversas reacciones, tanto cognitivas como conductuales, relacionadas con el sentimiento de tristeza. A nivel cognitivo, el ser humano se caracteriza por enfocar su atención en los síntomas depresivos, las causas de estos y sus consecuencias, así como el significado personal que se le asigna a dicho estado, y, por consiguiente, se evidencian patrones conductuales que no permiten la capacidad de reenfocar la atención hacia estímulos que no estén relacionados con el estado depresivo.

De acuerdo con lo anterior, Vázquez et al. (2010) sostienen que las personas que suelen con mayor regularidad tener rumiaciones depresivas, caracterizadas por pensamientos recurrentes relacionados con sus síntomas depresivos, o en las causas de estos y sus consecuencias, sufrirán durante más tiempo los efectos del estado de ánimo depresivo que aquellos que sean capaces de distraerse de los mismos.

Inoñán y Cassaretto (2011) plantean que estudios han encontrado que el estilo rumiativo influye directamente en el aumento de las atribuciones negativas y globales, como también en la desesperanza hacia 
el futuro (Lyubomirsky \& Nolen-Hoeksema, 1993). De igual manera, en la accesibilidad de recuerdos negativos (Lyumbomirsky, Caldwell, \& NolenHoeksema, 1998), el pesimismo y las interpretaciones negativas sesgadas (Lyubomirsky, Tucker, Caldwell, \& Berg, 1999), reduciendo la eficacia de la solución de problemas (Lyubomirsky y Nolen-Hoeksema, 1993) e incrementando la vulnerabilidad al desarrollo de sintomatología ansiosa (Nolen-Hoeksema, 2000).

Spasojevic y Alloy (2001) afirman que el estilo rumiativo media la relación de diversos factores de vulnerabilidad cognitiva y de personalidad, y que la aparición de nuevos casos de depresión a lo largo del tiempo, como también los sujetos que son propensos a tener un estilo atribucional depresógeno y baja autoestima, acompañado con estilos de pensamientos rumiativos, manifiestan mayor riesgo a sufrir episodios depresivos.

Vázquez, et al. (2010), de igual manera, muestra diversas explicaciones con respecto al origen de los estilos rumiativos. En primera instancia, menciona la teoría inicial de Nolen-Hoeksema (1991), quien sugería que el estilo rumiativo es aprendido mediante el modelamiento de figuras importantes. Posteriormente, Papageorgiou y Wells (2001), Watkins y Baracaia (2001) consideraron que el individuo, al percibir que las respuestas rumiativas eran útiles para su estado depresivo, influía en que se siguieran manteniendo. Por otra parte, otros estudios han propuesto que el estilo rumiativo podría estar relacionado con la vulnerabilidad a la depresión, a través de su relación con diferentes variables como el neuroticismo (Nolan, Roberts, \& Gotlib, 1998; Roberts, Gilboa, \& Gotlib, 1998), la tendencia a experimentar estados emocionales complejos (Hervás, \& Vázquez, 2006) y la alta sensibilidad al castigo (Leen-Feldner, Zvolensky, Feldner, \& Lejuez, 2004).

Es así como han surgido diversas teorías que han pretendido explicar cómo se desarrolla la vulnerabilidad cognitiva en un individuo y cómo esta lo predispone al desarrollo de patologías clínicas.

En el presente capitulo se realizó una revisión teórica, explicando todo lo concerniente al desarrollo de las estructuras cognitivas que cada individuo presenta, dependiendo de sus factores psicológicos, sociales y biológicos (experiencias tempranas aversivas, temperamento, etc.), para 
dar explicación al por qué algunas personas son más vulnerables que otras a desarrollar patologías, como, por ejemplo, la depresión.

Si es posible comprender cómo se desarrolla la vulnerabilidad cognitiva, que predispone al sujeto a una posibilidad mayor de adquirir un trastorno, se podrían diseñar distintas estrategias que promuevan estilos cognitivos saludables y disminuyan los factores de riesgo en la población.

\section{Referencias}

Abela, J.R.Z. (2002). Depressive mood reactions to failure in the achievement domain: a test of the integration of the hopelessness and self-esteem theories of depression. Cognitive Therapy and Research, 26(4), 531-552. https://doi.org/10.1023/A:1016236018858

Abramson, L.Y., Seligman, M.E.P., \& Teasdale, J.D. (1978). Learned helplessness in humans: critique and reformulation. Journal of Abnormal Psychology, 87(1), 49-74. http://dx.doi.org/10.1037/0021-843X.87.1.49

Alloy, L. B., Abramson, L. Y., \& Francis, E. L. (1999). Do negative cognitive styles confer vulnerability to depression?. Current Directions in Psychological Science, 8(4), 128-132. https://doi.org/10.1111/14678721.00030

Alloy, L. B., Abramson, L. Y., Walshaw, P. D., Gerstein, R. K., Keyser, J. D., Whitehouse, W. G., ... \& Harmon-Jones, E. (2009). Behavioral approach system (BAS)-relevant cognitive styles and bipolar spectrum disorders: Concurrent and prospective associations. Journal of Abnormal Psychology, 118(3), 459. https://doi.org/10.1037/ a0016604

Beck, A. (2012). Terapia cognitiva de la depresión; 20ª edición. Bilbao: Desclée de Brouwer.

Beck, J.S. (2000). Terapia cognitiva conceptos básicos y profundización. Barcelona, España: Editorial Gedisa.

Beevers, C. (2005). Cognitive vulnerability to depression: a dual process model. Clinical Psychology Review, 25(7), 975-1002. https://doi. org/10.1016/j.cpr.2005.03.003

De Raedt, R., \& Koster, E. H. W. (2010). Understanding vulnerability for depression from a cognitive neuroscience perspective: a reappraisal 
of attentional factors and a new conceptual framework. Cognitive, Affective, and Behavioral Neuroscience, 10(1), 50-70. https://doi. org/10.3758/CABN.10.1.50

Duque, A., López-Gómez, I., Blanco, I., \& Vásquez, C. (2015). Modificación de sesgos cognitivos (msc) en depresión. Una revisión crítica de nuevos procedimientos para el cambio de sesgos cognitivos. Revista Terapia Psicológica, 33 (2), 103-116. http://dx.doi.org/10.4067/ S0718-48082015000200005

Fresco, D.M., Rytwinsky, N.K., \& Craighead, L.W. (2007). Explanatory flexibility and negative life events interact to predict depression symptoms. Journal of Social and Clinical Psychology, 26(5), 595-608. http://dx.doi.org/10.1521/jscp.2007.26.5.595

Gotlib, I. H., \& Joormann, J. (2010). Cognition and depression: Current status and future directions. Annual Review of Clinical Psychology, 6, 285-312. https://doi.org/10.1146/annurev.clinpsy.121208.131305

Hammen, C. (1991). Generation of stress in the course of unipolar depression. Journal of

Abnormal Psychology, 100(4), 555-561. http://dx.doi.org/10.1037/0021-843X.100.4.555

Hervás, G., \& Vázquez, C. (2006). La regulación afectiva: modelos, investigación e implicaciones para la salud mental y física. Revista de Psicología General y Aplicada, 59(1-2), 9-32.

Ingram, R., Miranda, J., \& Segal, Z. (1998). Cognitive Vulnerability to depression. New York: Guilford Press.

Inoñan, C., \& Cassaretto, M. (2011). Propiedades psicométricas de la escala de respuestas rumiativas en una muestra de adultos de Lima. (Tesis de pregrado). Pontificia Universidad Católica del Perú. Recuperado de: http://tesis.pucp.edu.pe/repositorio/handle/123456789/1286

Kagan, J., \& Snidman, N. (2004). The Long Shadow of Temperament. Massachusetts: Belknap Press.

Leen-Feldner, E. W., Zvolensky, M. J., Feldner, M. T., \& Lejuez, C. W. (2004). Behavioral inhibition: relation to negative emotion regulation and reactivity. Personality and Individual Differences, 36(6), 1235-1247. https://doi.org/10.1016/S0191-8869(02)00113-7 
Londoño, N.H., Jiménez, E.B., Juárez, F, Marín, C.A. (2010). The components of cognitive vulnerability to generalized anxiety disorder. International Journal of Psychological Research, 3(2), 43-54. https:// doi.org/10.21500/20112084.811

Lyubomirsky, S., Caldwell, N., \& Nolen-Hoeksema, S. (1998). Effects of ruminative and distracting responses to depressed mood on retrieval of autobiographical memories. Journal of Personality and Social Psychology, 75(1), 166-177. http://dx.doi.org/10.1037/00223514.75.1.166

Lyubomirsky, S., \& Nolen-Hoeksema, S. (1993). Self-perpetuating properties of dysphoric rumination. Journal of Personality and Social Psychology, 65(2), 339-349. http://dx.doi.org/10.1037/00223514.65.2.339

Lyubomirsky, S., Tucker, K., Caldwell, N. D., \& Berg, K. (1999). Why ruminators are por problem solvers: Clues from the phenomenology of dysphoric rumination. Journal of Personality and Social Psychology, 77(5), 1041-1060. http://dx.doi.org/10.1037/00223514.77.5.1041

Martínez, C. (2011). Psicooncología e indefensión aprendida. (Tesis Doctoral). Universidad de Granada, España. Recuperado de: http://digibug.ugr.es/handle/10481/15463

Mathews, A., \& MacLeod, C. (2005). Cognitive vulnerability to emotional disorders. Annual Review of Clinical Psychology, 1, 167-195. https://doi.org/10.1146/annurev.clinpsy.1.102803.143916

Matt, G., Vázquez, C., y Campbell, K. (1992). Mood congruent recall of affectively toned stimuli: a meta-analytic review. Clinical Psychology Review, 12(2), 227-255. https://doi.org/10.1016/0272-7358(92)90116-P

McGonagle, K. A., \& Kessler, R. C. (1990). Chronic stress, acute stress, and depressive symptoms. American journal of community psychology, 18(5), 681-706. https://doi.org/10.1007/BF00931237

Moor, M., \& Fresco, D. M (2007). The relationship of explanatory flexibility to explanatory style. Behavior Therapy, 38(4), 325-332. https:// doi.org/10.1016/j.beth.2006.06.007 
Nolan, S., Roberts, J., \& Gotlib, I. (1998). Neuroticism and Ruminative Response Style as Predictors of Change in Depressive Symptomatology. Cognitive Therapy \& Research, 22(5), 445-455. https://doi. org/10.1023/A:1018769531641

Nolen-Hoeksema, S. (2000) The role of rumination in depressive disorders and mixed anxiety/depressive symptoms. Journal of Abnormal Psychology, 109(3), 504-511. http://dx.doi.org/10.1037/0021-843X.109.3.504

Nolen-Hoeksema, S. (1991) Responses to Depression and Their Effects on the Duration of Depressive Episodes. Journal of Abnormal Psychology, 100(4), 569-582. http://dx.doi.org/10.1037/0021-843X.100.4.569

Papageorgiou, C. \& Wells, A. (2001). Positive beliefs about depressive rumination: development and preliminary validation of a self-report scale. Behavior Therapy, 32(1), 13-26. https://doi.org/10.1016/ S0005-7894(01)80041-1

Riso, W. (2009). Terapia cognitiva. Fundamentos teóricos y conceptualización del caso clínico. Barcelona, España: Editorial Paidós Ibérica.

Roberts, J., Gilboa, E. \& Gotlib, I. (1998) Ruminative Response Style and Vulnerability to Episodes of Dysphoria: Gender, Neuroticism, and Episode Duration. Cognitive Therapy and Research, 22(4), 401423. https://doi.org/10.1023/A:1018713313894

Spasojevic, J., y Alloy, L. (2001) Rumination as a Common Mechanism Relating Depressive Risk Factors to Depression. Emotion, 1(1), 25-37. http://dx.doi.org/10.1037/1528-3542.1.1.25

Turner, R. J., Wheaton, B., \& Lloyd, D. A. (1995). The epidemiology of social stress. American sociological review, 60(1) 104-125. http:// dx.doi.org/ 10.2307/2096348

Vanegas, H., Krouse, M., Bauer, S., Moessner, M., Espinoza, D., Carrasco, A., Botero, L., ..., \& Patiño, J. (2017). Uso de e-mental health para el seguimiento posterapia de pacientes con depresión en Colombia. Revista Virtual Universidad Catolica de Chile, 52, 41-56. Recuperado de: http://revistavirtual.ucn.edu.co/index.php/RevistaUCN/ article/view/943/1390 
Vázquez, C, Hervas, G., Hernangómez, L., \& Romero, N. (2010). Modelos cognitivos de la depresión síntesis y propuesta basada en 30 años de investigación. Psicología Conductual, 18(1), 139-165.

Vázquez, C., Díez-Alegría, C., Hernández-Lloreda, M.J., \& Nieto, M. (2008). Implicit and explicit self-schema in active deluded, remitted deluded, and depressed patients. Journal of Behavior Therapy and Experimental Psychiatry, 39(4), 587-99. https://doi.org/10.1016/j. jbtep.2008.01.006

Watkins, E., \& Baracaia, S. (2001). Why do people ruminate in dysphoric moods? Personality and Individual Differences, 30(5), 723-734. http://dx.doi.org/10.1016/S0191-8869(00)00053-2

Williams, J. M. G., Barnhofer, T., Crane, C., Hermans, Raes, F., Watkins, Ed. \& Dalgleish, T. (2007). Autobiographical memory specificity and emotional disorder. Psychological Bulletin, 133(1), 122-148. https://dx.doi.org/10.1037\%2F0033-2909.133.1.122

Whitmer, A. J., \& Gotlib, I. H. (2013). An attentional scope model of rumination. psychological Bulletin, 139(5), 1036-1061. http://dx.doi. org/10.1037/a0030923

Young, J., Klosko, J. S., \& Weishaar, M. E. (2013). Terapia de esquemas guía práctica. Bilbao: Desclée de Brouwer. 